\title{
COMPONENTES FISIOLÓGICOS E CRESCIMENTO INICIAL DE MUDAS DE PALMEIRA- GARRAFA EM RESPOSTA A SUBSTRATOS COM LODO DE CURTUME
}

\author{
Physiological components and initial growth seedlings of palm tree-bottle in response to substrates with tannery sludge
}

Sávio da Silva Berillii ${ }^{1}$ Ramon Amaro de Sales²*; Ana Paula Braido Pinheiro ${ }^{3}$; Lucas Celim Pereira ${ }^{4}$; Luis Eduardo Gottardo ${ }^{4}$; Ana Paula Candido Gabriel Berilli ${ }^{1}$

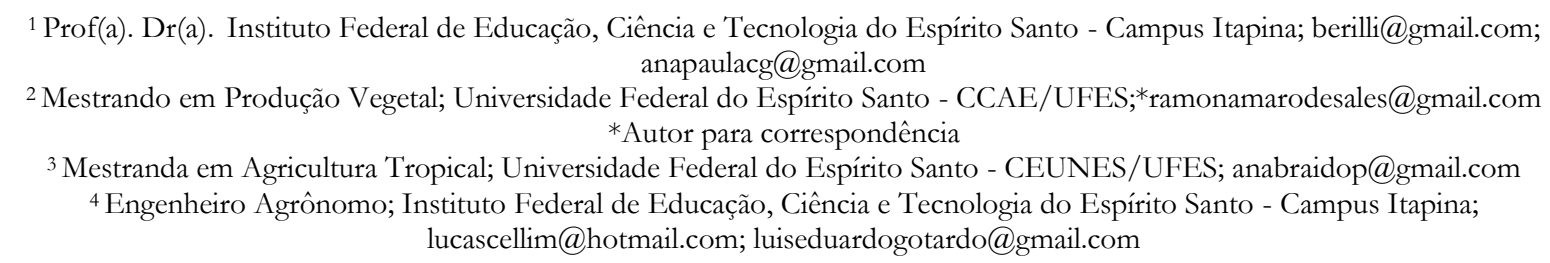

Artigo enviado em 24/08/2017, aceito em 07/03/2018 e publicado em 10/04/2018.

Resumo - As plantas ornamentais são importantes na cultura e no bem estar da população e por isso, possui grande mercado na sociedade brasileira e mundial. A produção de mudas de espécies ornamentais sofisticadas e de crescimento lento, como é o caso da palmeira garrafa, associada ao apelo sustentável de reutilização de resíduos industriais, como é o caso do lodo de curtume em substrato, pode ganhar relevante importância para compradores desse tipo de planta, desde que esse resíduo não comprometa o crescimento e a fisiologia da muda. O objetivo do trabalho foi avaliar o efeito de diferentes proporções de lodo de curtume desidratado adicionado ao substrato no desenvolvimento e fisiologia de mudas de palmeira-garrafa. O experimento foi conduzido em delineamento inteiramente casualizado com quatro repetições e seis tratamentos, sendo um substrato comercial utilizado pelos produtores, um tratamento contendo apenas solo e os outros quatro com diferentes proporções de lodo de curtume desidratado $(10,20,30$, e 40\%), misturados ao solo. Avaliou-se nesse experimento as características de desenvolvimento e fisiologia das mudas. Os tratamentos com $10 \%$ de lodo de curtume e o tratamento convencional apresentaram os melhores resultados para as características de desenvolvimento. Quanto a fisiologia, o substrato convencional foi melhor, com um maior balanço de nitrogênio, enquanto os tratamentos com lodo de curtume tiveram maiores índices de flavonoides, indicando maior estresse dessas mudas.

Palavras-chave - Hyophorbe lagenicaulis, paisagismo, sustentabilidade.

\begin{abstract}
Ornamental plants are important in the culture and well-being of the population and therefore, it has a large market in Brazilian and world society. The production of seedlings of sophisticated and slow-growing ornamental species, such as the palm tree bottle, associated with the sustainable use of industrial waste reuse, such as tanning sludge in substrate, may gain relevant importance for buyers of this type As long as this residue does not compromise the growth and physiology of molt. The objective of this work was to evaluate the effect of different proportions of dehydrated tannery sludge added to the substrate in the development and physiology of palm tree seedlings. The experiment was conducted in a completely randomized design with four replicates and six treatments, a commercial substrate used by the producers, a treatment containing only soil and the other four with different proportions of dehydrated tanning sludge (10, 20, 30 and 40\%), Mixed with the soil. The developmental and physiological characteristics of the seedlings were evaluated in this experiment. The treatments with $10 \%$ of tannery sludge and the conventional treatment presented the best results for the developmental characteristics. As for physiology, the conventional substrate was better, with a higher nitrogen balance, while treatments with tannery sludge had higher rates of flavonoids, indicating higher stress of these seedlings.
\end{abstract}

Keywords - Hyophor belagenicaulis, landscaping, sustainability. 


\author{
REVISTA SCIENTIA AGRARIA \\ Versão On-line ISSN 1983-2443 \\ Versão Impressa ISSN 1519-1125 \\ SA vol. $19 \mathrm{n}^{\circ} .1$ Curitiba Jan/Mar 2018 p. 94-101
}

\section{INTRODUÇÃO}

A Hyophorbe lagenicaulis, vulgarmente chamada de palmeira-garrafa, pertence à família Arecaceae (Palmae), sendo ela originaria das Ilhas Mascarenhas no Oceano Índico, no qual seus frutos possuem um alto potencial fisiológico para sobreviver em solos secos (DRANSFIELD et al., 2008; ASMUSSEN-LANGE; MAUNDER.; FAY, 2011). Segundo Matthes e Uzzo (2010), por ser uma planta de baixo porte, a palmeiragarrafa apresenta vantagens em densidade de plantio, comparado com outras palmeiras de médio e grande porte e, ainda, permite um melhor manejo devido a esta característica.

Para fins paisagísticos algumas práticas devem ser levadas em consideração, destacando-se a produção de mudas vigorosas e sadias, que aumentam a taxa de "pegamento" no campo e consequentemente reduzem gastos com replantio. Nesse contexto, o substrato precisa garantir ás mudas um bom desenvolvimento do sistema radicular e da parte aérea, visto que o mesmo está relacionado com a nutrição, aeração e capacidade de retenção de umidade do solo (BELTRÃO; OLIVEIRA, 2011; SOARES et al., 2016).

Um grande desafio das empresas geradoras de resíduos sólidos está em sua destinação final, sendo muitas vezes descartados em locais inapropriados. Neste contexto, uma alternativa excelente paradestinar esseresíduo seria reutilizá-lo na própria agricultura,garantido assim a sustentabilidade da relação solo-planta (BERILLI et al., 2017).

Deste modo, o lodo de curtume é um biofertilizante oriundo das indústrias curtumeiras com grande potencial de uso na agricultura, visto que apresenta alto teor de matéria orgânica e elementos minerais essenciais às plantas como o nitrogênio, $\mathrm{O}$ cálcio, o potássio dentre outros. Diversos autores estão pesquisando o uso deste resíduo na agricultura nas mais diferentes espécies, a fim de utiliza-lo como fonte alternativa em substratos (SALES et al., 2016; SALES et al., 2017; Quartezani et al., 2018; Berilli et al., 2018).

Segundo Berilli et al. (2015), a utilização de resíduos orgânicos industriais, como o lodo de curtume, constitui uma alternativa na adubação, com redução dos custos e consequente aumento da viabilidade econômica para produtores e indústrias. Além disso, o aproveitamento desses resíduos oriundos das indústrias cortumeiras, pode diminuir o impacto ambiental gerado pelo descarte desse subproduto ao meio ambiente.

Pesquisas com utilização de resíduos orgânicos têm demonstrado resultados relevantes em mudas de palmeiras, como na espécie Euterpe edulis Martius com lodo de esgoto compostado e cascas da palmeira pupunha (Silva et al., 2015) e com o resíduo de casca de arroz carbonizada para as espécies Euterpe edulis eRoystonea regia (BRAHM et al., 2013), entretanto, pouco se sabe sobre o efeito do lodo de curtume em espécies de palmeiras.

No paisagismo observa-se a busca por plantas vigorosas e saudáveis com a finalidade de melhor harmonizar o ambiente, portanto, a produção de mudas robustas e com boa aparência são cada vez mais procuradas para fins ornamentais. Com isso, a utilização do lodo de curtume pode ser uma alternativa viável para a produção de Hyophorbe lagenicaulis, uma vez que o uso de biofertilizantes têm proporcionado melhorias nas qualidades químicas, físicas e biológicas do solo, podendo minimizar impactos indesejáveis ao meio ambiente provocados por determinados adubos minerais industrializados (NAZARIO;MIOTTI; SOUZA, 2013).

Deste modo, com o intuito de reaproveitar os resíduos produzidos pelas indústrias de tratamento de couro, este trabalho objetivou avaliar o desenvolvimento e de mudas da palmeira-garrafa (Hyophorbe lagenicaulis), em substratos com variadas proporções de lodo de curtume e solo, assim como seus efeitos nos índices de compostos secundários, clorofilas e balanço de nitrogênio dessas mudas.

\section{MATERIAL E MÉTODOS}

O ensaio foi realizado no Instituto Federal de Educação, Ciência e Tecnologia do Espírito Santo Campus Itapina, localizado no município de Colatina, com coordenadas geográficas de $19^{\circ} 32^{\prime} 22^{\prime \prime}$ de latitude sul; $40^{\circ} 37^{\prime} 50^{\prime \prime}$ de longitude oeste e altitude de 71 metros. Foi conduzido em viveiro de propagação de mudas com sombrite $50 \%$ e irrigação por nebulização, deixando o substrato sempre na capacidade de campo, utilizando sementes de palmeira-garrafa (Hyophorbe lagenicaulis).

O delineamento experimental usado foi o inteiramente casualizado com quatro repetições e seis tratamentos. Os tratamentos seguem detalhados: T-0: 100\% solo; T-10: Uma mistura na proporção de $90 \%$ de solo $+10 \%$ de lodo de curtume desidratado; T-20: Uma mistura na proporção de $80 \%$ de solo $+20 \%$ de lodo de curtume desidratado; T-30: Uma mistura na proporção de $70 \%$ de terra $+30 \%$ de lodo de curtume desidratado; T-40: Uma mistura na proporção de $60 \%$ de terra $+40 \%$ de lodo de curtume desidratado; T-C: Uma mistura de solo + esterco bovino curtido na proporção de $3: 1+5 \mathrm{~kg} \cdot \mathrm{m}^{-3}$ de superfosfato simples + $1 \mathrm{~kg} \cdot \mathrm{m}^{-3}$ de $\mathrm{KCl}$ (convencional). O solo utilizado para as misturas dos substratos é classificado como um Latossolo Vermelho Distrófico (EMBRAPA, 2013) com suas caracteristicas descritas na Tabela 1: 
Tabela 1. Características químicas do solo usado como componente do substrato das mudas.

\begin{tabular}{|c|c|c|c|c|c|c|c|c|c|c|c|c|c|c|c|c|c|c|c|}
\hline \multirow[t]{2}{*}{$\mathrm{pH}$} & $\mathrm{P}$ & K & $\mathrm{Ca}$ & $\mathrm{Mg}$ & $\mathrm{Al}$ & $\mathrm{Na}$ & \multirow{2}{*}{$\begin{array}{l}\mathrm{C} \\
\%\end{array}$} & \multirow{2}{*}{$\begin{array}{l}\mathrm{MO} \\
\mathrm{g} / \mathrm{dm}^{3}\end{array}$} & SB & $\mathrm{T}$ & $\mathrm{T}$ & \multirow{2}{*}{$\begin{array}{l}\mathrm{m} \\
\%\end{array}$} & \multirow[t]{2}{*}{ V } & \multirow{2}{*}{$\mathrm{Fe}$} & \multicolumn{3}{|c|}{$\mathrm{Cu} \quad \mathrm{Zn} \quad \mathrm{Mn}$} & \multirow{2}{*}{ S } & $\mathrm{B}$ \\
\hline & $-\mathrm{m}$ & $\mathrm{dm}^{3-}$ & - & $\mathrm{cmol}_{\mathrm{c}}$ & $/ \mathrm{dm}$ & --- & & & $--\mathrm{cm}$ & $\mathrm{l}_{\mathrm{c}} / \mathrm{d}$ & $m^{3}--$ & & & & & $\mathrm{mg} /$ & $\mathrm{Im}^{3}-$ & & \\
\hline 5 & 5 & 48 & 0,8 & 1,3 & 0 & 0,03 & 47 & 8,1 & 2,3 & 3,1 & 2,3 & 0 & 74 & 7 & 0,6 & 0,8 & 7,9 & 112 & 0,4 \\
\hline
\end{tabular}

O lodo utilizado foi cedido pela empresa Capixaba Couros LTDA ME de Baixo Guandu-ES. Antes do estudo e avaliação da aptidão agronômica do composto na propagação da palmeira-garrafa, foram realizadas amostras e posteriormente análises, objetivando a caracterização completa do material e atendimento a Instrução Normativa $\mathrm{n}^{\circ} 27$, de 05 de junho de 2006 (MAPA, 2006), que dispõe sobre os fertilizantes, corretivos, inoculantes e biofertilizantes, e que impõe limites sobre os valores máximos admitidos, quanto à microbiologia, sanidade, fitossanidade, e metais pesados, para possível registro e comercialização.

O composto de lodo decurtume apresentou em sua composição: Carbono Orgânico 61,2 g.dm³ Relação C/N 3/1; Matéria Orgânica Total 293,6 g.dm³; Matéria Orgânica Compostável 110,2 g.dm³ Nitrogênio 17,6 g.dm³ ; Fósforo 2,27 g.dm³ ; Potássio 0,49 g.dm³ Cálcio 225,9 g. $\mathrm{dm}^{3}$; Magnésio 15,6 g.dm³ Enxofre 13,2 g.dm³ ; Boro 25,8 g.dm³; Sódio 13,2 g. $\mathrm{dm}^{3}$; Cromo 37,0 g. $\mathrm{dm}^{3}$.

As sementes foram adquiridas em viveiro comercial de propagação de mudas da região de Camaçari na Bahia (Viveiro Orquilândia), sendo estas, já no IFES-Itapina, colocadas para germinar em canteiros de areia lavada, onde permaneceram durante 12 meses, atingindo altura média de $10 \mathrm{~cm}$, quando foi contabilizado o índice de germinação das sementes. Após esse período, as mudas foram transplantadas para sacos de polietileno com dimensões de $18 \times 24 \mathrm{~cm}$, dentro do delineamento programado. Realizou-se o controle das plantas daninhas durante toda a condução do experimento.

A etapa de propagação e aplicação dos tratamentos durou 26 meses após o replantio, tempo este em que as plantas já apresentavam porte adequado para serem transplantadas para local definitivo. Ao fim do experimento, mensuraram-se as seguintes características de desenvolvimento: diâmetro do colo $(\mathrm{mm})$; altura da roseta $(\mathrm{cm})$; altura da planta $(\mathrm{cm})$; diâmetro da roseta $(\mathrm{mm})$; diâmetro da copa $(\mathrm{cm})$; e número de folhas.

Para as análises de cor foi utilizado um colorímetro modelo CR-400 (Konica Minolta), onde as características de luminosidade (L) e cromaticidade (a, b e $b^{0}$ em configuração DL/65) foram medidas nas folhas da palmeira-garrafa em duas estratificações da copa, sendo uma análise da primeira folha completamente expandida e uma da segunda folha completamente expandida.

Nesse mesmo período, na parte aérea das mudas, foi feita uma análise com um fluorômetro modelo Multiplex ${ }^{\circledR}$ (Force-A), com fontes múltiplas de excitação de luz (ultravioleta, azul, verde e vermelho), estimando índices de vários compostos, como balanço de nitrogênio (NBI-G e NBI-R), clorofila (SFR-G e SFR-R), antocianina (ANT-RG e ANT-RB) e flavonoides (FLAV). Os índices obtidos pelo Multiplex ${ }^{\circledR}$ por vezes, possuem mais de um por característica, derivados das diferentes combinações de comprimentos de ondas emitidos pelo equipamento. Todas as avalições foram realizadas no período da manhã, entre as 9:00 e 11:00 horas e em apenas um lado das mudas, apontando o equipamento para a copa, de cima para baixo, em um ângulo aproximadamente de 45 graus e os dados obtidos foram parametrizados.

Os dados foram submetidos à análise de variância, pelo teste $\mathrm{F}$, e havendo significância, as médias foram comparadas pelo teste de Dunnett a $5 \%$ de probabilidade. Foram ajustados modelos de regressão para as proporções de lodo de curtume, quando o efeito foi significativo pelo teste $\mathrm{F}$, e toda a estatística foi realizada com auxílio do programa estatístico R (R Core Team, 2016).

\section{RESULTADOS E DISCUSSÃO}

As mudas apresentaram crescimento lento, típico da espécie testada, além de um baixo índice de germinação, com 52\% de pegamento. Através dos dados obtidos por este experimento na Tabela 2, percebe-se que o tratamento convencional obteve melhores valores médios para as caracteristicas de desenvolvimento, seguido pelo tratamento com $10 \%$ de lodo de curtume que foi inferior apenas no ganho em altura de planta.

Para a característica número de folhas e diâmetro da roseta, não foi encontrado diferença entre os tratamentos, destacando a potencialidade desse resíduo alternativo no ganho dessas caracteristicas quando comparado ao tratamento convencional. Os autores Martins Filho et al. (2007), encontraram influência do substrato no número de folhas em palmeira pupunheira utilizando nove substratos, contrastando com os resultados deste experimento, visto que não houve diferença significativa. 
Para a caracteristica diâmetro de colo, o tratamento com $10 \%$ de lodo de curtume apresentou resultado igual ao tratamento convencional. No entanto, com o aumento das proporções de lodo de curtume no substrato, os resutados se diferem, sendo inferiores ao tratamento convencional juntamente com o T-0, que utilizou apenas solo como substrato.

Quando avaliado a altura da roseta (Tabela 2), percebe-se que independente da concentração de lodo de curtume utilizado, o resultado foi igual ao tratamento convencional. Isso pode estar ligado aos benefícios nutricionais que o lodo possui, como o fornecimento de diversos nutrientes. Todavia, o tratamento T-0 apresentou resultado inferior ao tratamnto convencional, apresentando uma redução de mais de $70 \%$ para essa caracteristica.
Para a altura de planta, é notório que o melhor resultado foi no tratamento convencional, sendo que, de acordo com o aumento das doses de lodo de curtume no substrato, ocorreu uma redução gradativa na altura da planta. Essa diminuição da altura da palmeira-garrafa pode estar atrelada ao elemento cromo e sódio, que nas concentrações oferecidas pelo lodo de curtume (Sódio 13,2 g. $\mathrm{dm}^{3}$ e Cromo 37,0 g. $\mathrm{dm}^{3}$ ) são tóxicas as plantas e, com o aumento da concentração deste composto, mais deletério foram seus efeitos, sendo o mesmo comportamento observado no experimento de Berilli et al. (2014), na altura de plantas de mudas de café conilon com doses crescentes de lodo de curtume.

Tabelas 2. Valores médios de diâmetro do colo (DC), diâmetro da roseta, altura da roseta (AR), diâmetro da copa (DCO), número de folhas (NF) e altura de planta (AP) de mudas de palmeira cultivadas em substrato convencional e com diferentes concentrações de lodo de curtume desidratado.

\begin{tabular}{lllllll}
\hline Tratamento & DC $(\mathrm{mm})$ & AR $(\mathrm{cm})$ & AP $(\mathrm{cm})$ & NF & DCO $(\mathrm{cm})$ & DR $(\mathrm{mm})$ \\
\hline T-C & 44,09 & 13,50 & 54,50 & 3,5 & 55,33 & 24,91 \\
T-0 & $25,20^{*}$ & $7,75^{*}$ & $34,25^{*}$ & $3,0^{\mathrm{n} / \mathrm{s}}$ & $41,83^{*}$ & $25,50^{\mathrm{n} / \mathrm{s}}$ \\
T-10 & $34,87^{\mathrm{n} / \mathrm{s}}$ & $11,50^{\mathrm{n} / \mathrm{s}}$ & $45,33^{*}$ & $3,0^{\mathrm{n} / \mathrm{s}}$ & $48,75^{\mathrm{n} / \mathrm{s}}$ & $25,59^{\mathrm{n} / \mathrm{s}}$ \\
T-20 & $30,03^{*}$ & $10,66^{\mathrm{n} / \mathrm{s}}$ & $44,67^{*}$ & $4,0^{\mathrm{n} / \mathrm{s}}$ & $47,33^{\mathrm{n} / \mathrm{s}}$ & $27,88^{\mathrm{n} / \mathrm{s}}$ \\
T-30 & $29,12^{*}$ & $10,75^{\mathrm{n} / \mathrm{s}}$ & $44,00^{*}$ & $3,5^{\mathrm{n} / \mathrm{s}}$ & $45,83^{\mathrm{n} / \mathrm{s}}$ & $24,74^{\mathrm{n} / \mathrm{s}}$ \\
T-40 & $27,70^{*}$ & $9,75^{\mathrm{n} / \mathrm{s}}$ & $42,12^{*}$ & $4,0^{\mathrm{n} / \mathrm{s}}$ & $44,50^{*}$ & $25,85^{\mathrm{n} / \mathrm{s}}$ \\
\hline Média & 31,84 & 10,65 & 44,14 & 3,5 & 47,26 & 25,74 \\
\hline CV $(\%)$ & 22,24 & 20,55 & 9,12 & 25,2 & 11,10 & 18,06 \\
\hline
\end{tabular}

Médias seguidas por * diferem estatisticamente do tratamento convencional pelo Teste de Dunnett ao nível de 5\% de probabilidade. n/s: não significativo.

Para a característica diâmetro de copa (Tabela 2), os tratamentos com 10, 20 e 30\% de lodo de curtume no substrato foram iguais ao tratamento convencional. O mesmo comportamento não é observado quando aumentado essa proporção para $40 \%$, no qual o resultado difere do convencional, com uma redução de $24 \%$ para essa característica.

Pelas análises de regressão, foi observado que somente a altura de planta (Figura 1) apresentou significância, sendo constatada uma equação de segundo grau e que, ao estimar a dose ótima de lodo de curtume, o mesmo foi obtido com $24,55 \%$, resultando em um crescimento de $45,36 \mathrm{~cm}$ de altura.

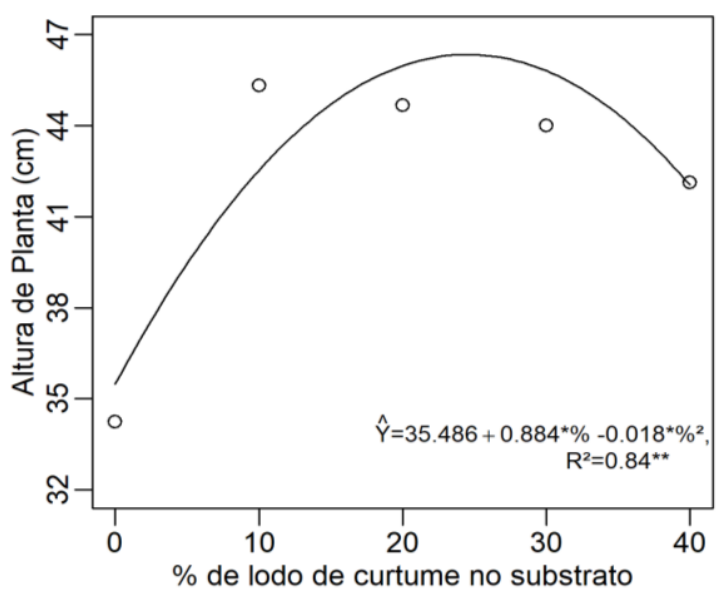

Figura 1. Comportamento da variávelaltura de planta em função de diferentes proporções de lodo de curtume no substrato de mudas de palmeira-garrafa. $* * 1 \%$ de probabilidade. 
A Tabela 3 apresenta as variáveis obtidas pelo aparelho Multiplex ${ }^{\circledR}$, no qual não foi observada diferença para os índices de antocianinas e de clorofila estimada pela luz vermelha (SFR-R). O índice de clorofila estimado pela luz verde (SFR-G) apresentou diferença, sendo observado somente no T-0, tratamento este que utilizou apenas solo, apresentando uma redução de 40\% em suas clorofilas quando comparado ao tratamento convencional.

Essa redução se deve principalmente pela menor quantidade de nutrientes que este tratamento apresentou, visto que o tratamento convencional recebeu além da matéria orgânica proveniente de esterco bovino, uma adição de $5 \mathrm{~kg} \cdot \mathrm{m}^{3}$ de superfosfato simples e $1 \mathrm{~kg} \cdot \mathrm{m}^{3}$ de $\mathrm{KCl}$. Para os índices de flavonóides e balanço de nitrogênio (Tabela 3), é observado que todos os tratamentos foram diferentes do tratamento convencional. Quanto à produção de flavonóides, ao comparar as diferentes proporções de lodo de curtume com o tratamento convencional, observa-se um aumento na biossíntese deste composto de $15,21,21$ e $23 \%$ nos respectivos tratamentos $\mathrm{T}-10$, T-20, T-30 e T-40.

Os autores Berilli et al. (2016), também encontraram aumento da produção de flavonóides em resposta a substratos com lodo de curtume, revelando que a presença desse resíduo promove aumento na produção de compostos secundários como os flavonóides. Segundo Kandil et al. (2004), plantas que sofrem estresse tendem a produzir uma maior quantidade de polifenóis, como flavonóides e taninos, sendo que os elementos cromo e o sódio, por estarem em excesso, podem ter causado esse estresse para essas mudas, ocasionando o aumento deste composto secundário. Apesar disso, os índices de antocianinas (ANTH-RG e ANTH-RB) não foram alterados com a presença do lodo de curtume, somente os índices de flavonoides.

Tabela 3. Índices médios de flavonóides, antocianina, clorofila e balanço de nitrogênio, obtidas utilizando o equipamento o Multiplex ${ }^{\circledR}$ em mudas de palmeira-garrafa, cultivadas em substrato convencional e com diferentes concentrações de lodo de curtume desidratado.

\begin{tabular}{llllllll}
\hline Tratamento & FLAV & NBI-R & NBI-G & SFR-R & SFR-G & ANTH-RG & ANTH-RB \\
\hline T-C & 0,92 & 0,22 & 0,20 & 1,80 & 2,29 & $-0,14$ & $-0,72$ \\
T-0 & $1,11^{*}$ & $0,10^{*}$ & $0,12^{*}$ & $1,77^{\mathrm{n} / \mathrm{s}}$ & $1,63^{*}$ & $-0,03^{\mathrm{n} / \mathrm{s}}$ & $-0,68^{\mathrm{n} / \mathrm{s}}$ \\
T-10 & $1,06^{*}$ & $0,14^{*}$ & $0,13^{*}$ & $1,67^{\mathrm{n} / \mathrm{s}}$ & $1,73^{\mathrm{n} / \mathrm{s}}$ & $-0,11^{\mathrm{n} / \mathrm{s}}$ & $-0,62^{\mathrm{n} / \mathrm{s}}$ \\
T-20 & $1,12^{*}$ & $0,15^{*}$ & $0,14^{*}$ & $1,86^{\mathrm{n} / \mathrm{s}}$ & $2,05^{\mathrm{n} / \mathrm{s}}$ & $-0,08^{\mathrm{n} / \mathrm{s}}$ & $-0,69^{\mathrm{n} / \mathrm{s}}$ \\
T-30 & $1,12^{*}$ & $0,13^{*}$ & $0,12^{*}$ & $1,69^{\mathrm{n} / \mathrm{s}}$ & $2,05^{\mathrm{n} / \mathrm{s}}$ & $-0,09^{\mathrm{n} / \mathrm{s}}$ & $-0,63^{\mathrm{n} / \mathrm{s}}$ \\
T-40 & $1,14^{*}$ & $0,15^{*}$ & $0,14^{*}$ & $2,08^{\mathrm{n} / \mathrm{s}}$ & $2,21^{\mathrm{n} / \mathrm{s}}$ & $-0,08^{\mathrm{n} / \mathrm{s}}$ & $-0,66^{\mathrm{n} / \mathrm{s}}$ \\
\hline Média & 1,08 & 0,15 & 0,14 & 1,81 & 1,99 & $-0,09$ & $-0,67$ \\
CV (\%) & 7,72 & 16,85 & 16,29 & 20,41 & 14,84 & 16,68 & 13,07 \\
\hline
\end{tabular}

Médias seguidas por * diferem estatisticamente do tratamento convencional pelo Teste de Dunnett ao nível de 5\% de probabilidade. n/s: não significativo. Flavonóides (FLAV), antocianina (ANT-RG e ANT-RB), clorofila (SFR-G e SFR-R) e balanço de nitrogênio (NBI-G e NBI-R).

$\mathrm{Na}$ Tabela 4, observando os valores das características lidas pelo colorímetro, no que se refere à Folha 1, não apresentou diferença estatística entre os tratamentos para nenhuma característica avaliada, demonstrando que uma folha desse estádio de desenvolvimento e maturidade fisiológica não apresenta grandes variações de cor, mesmo submetidas a forte influência da aplicação dos substratos representadas pelos tratamentos. No entanto, a Folha 2, por ser uma folha mais velha e madura fisiologicamente, apresentou um padrão de resposta diferente da folha 1 , apontando diferenças entre os tratamentos para as variáveis analisadas.
O ângulo hue $\left(b^{0}\right)$ diferiu estatisticamente na Folha 2 somente para o T-0, tratamento esse que utilizou apenas solo de barranco, apresentando valores mais próximos do ângulo de $90^{\circ}$, revelando uma tendência de folhas mais cloróticas. Esse amarelecimento pode ser explicado pela mobilidade do nitrogênio na planta, visto que é um nutriente móvel na planta, translocando esse elemento de folhas mais velhas para folhas mais novas, o que pode explicar o porquê isso não ocorreu na primeira folha (BORIN et al., 2013).

Para o parâmetro de cromaticidade "a" e L da folha 2, não houve diferença, enquanto que $\mathrm{O}$ 
parâmetro " $b$ " apresentou diferença. O tratamento T-0 apresentou maiores valores de "b" quando comparadas ao tratamento convencional, inferindo que essas plantas apresentaram uma coloração mais amarela, provavelmente pela escassez de nutrientes deste tratamento. Esse fato revela que a adição do lodo de curtume promoveu algum grau de adubação às mudas, superior ao solo puro, pois mesmo sem adubação ou outra fonte de matéria orgânica, houve similaridade com o tratamento convencional em ambas as folhas avaliadas, quanto à coloração.

Tabela 4. Valoresmédios do índice de luminosidade (L), parâmetros de cromaticidade "a" e "b" e ângulo hue ( $h$ " $)$ em mudas de palmeira-garrafa cultivadas em substrato convencional e com diferentes concentrações de lodo de curtume desidratado.

\begin{tabular}{lllll|llll}
\hline & Folha 1 & \multicolumn{1}{l}{} & \multicolumn{4}{c}{ Folha 2} \\
\hline Trat & $\mathrm{L}$ & $\mathrm{a}$ & $\mathrm{b}$ & $b^{0}$ & $\mathrm{~L}$ & $\mathrm{a}$ & $\mathrm{b}$ & $b^{0}$ \\
\hline T-C & 32,9 & $-8,1$ & 11,3 & 120,6 & 35,1 & $-8,09$ & 12,4 & 117,9 \\
T-0 & $35,1^{\mathrm{n} / \mathrm{s}}$ & $-8,4^{\mathrm{n} / \mathrm{s}}$ & $13,1^{\mathrm{n} / \mathrm{s}}$ & $118,2^{\mathrm{n} / \mathrm{s}}$ & $40,5^{\mathrm{n} / \mathrm{s}}$ & $-6,96^{\mathrm{n} / \mathrm{s}}$ & $18,6^{*}$ & $108,8^{*}$ \\
T-10 & $34,0^{\mathrm{n} / \mathrm{s}}$ & $-8,4^{\mathrm{n} / \mathrm{s}}$ & $12,2^{\mathrm{n} / \mathrm{s}}$ & $119,8^{\mathrm{n} / \mathrm{s}}$ & $38,2^{\mathrm{n} / \mathrm{s}}$ & $-7,19^{\mathrm{n} / \mathrm{s}}$ & $12,9^{\mathrm{n} / \mathrm{s}}$ & $115,4^{\mathrm{n} / \mathrm{s}}$ \\
T-20 & $33,1^{\mathrm{n} / \mathrm{s}}$ & $-8,4^{\mathrm{n} / \mathrm{s}}$ & $11,8^{\mathrm{n} / \mathrm{s}}$ & $120,1^{\mathrm{n} / \mathrm{s}}$ & $33,4^{\mathrm{n} / \mathrm{s}}$ & $-7,36^{\mathrm{n} / \mathrm{s}}$ & $10,9^{\mathrm{n} / \mathrm{s}}$ & $116,8^{\mathrm{n} / \mathrm{s}}$ \\
T-30 & $30,5^{\mathrm{n} / \mathrm{s}}$ & $-6,7^{\mathrm{n} / \mathrm{s}}$ & $9,3^{\mathrm{n} / \mathrm{s}}$ & $121,6^{\mathrm{n} / \mathrm{s}}$ & $35,7^{\mathrm{n} / \mathrm{s}}$ & $-6,72^{\mathrm{n} / \mathrm{s}}$ & $12,2^{\mathrm{n} / \mathrm{s}}$ & $114,1^{\mathrm{n} / \mathrm{s}}$ \\
T-40 & $31,9^{\mathrm{n} / \mathrm{s}}$ & $-7,9^{\mathrm{n} / \mathrm{s}}$ & $10,8^{\mathrm{n} / \mathrm{s}}$ & $121,5^{\mathrm{n} / \mathrm{s}}$ & $33,9^{\mathrm{n} / \mathrm{s}}$ & $-7,15^{\mathrm{n} / \mathrm{s}}$ & $11,3^{\mathrm{n} / \mathrm{s}}$ & $115,4^{\mathrm{n} / \mathrm{s}}$ \\
\hline Média & 32,9 & $-8,0$ & 11,4 & 120,3 & 36,2 & $-7,2$ & 13,1 & 114,7 \\
CV $(\%)$ & 10,1 & 17,5 & 23,5 & 2,8 & 12,1 & 12,2 & 18,3 & 1,89 \\
\hline
\end{tabular}

Médias seguidas por * diferem estatisticamente do tratamento convencional pelo Teste de Dunnett ao nível de 5\% de probabilidade. n/s: não significativo.

Quando comparado as Folhas 1 e 2 é observado ainda que a Folha 1 apresentou valor mais negativo de "a", indicando coloração mais esverdeada. A Folha 2 apresentou maior média de "b", inferindo coloração mais amarela para essas folhas, juntamente com o ângulo bue mais próximo de $90^{\circ}$. Com isso, percebe-se que houve diferença no grau de maturidade das folhas, visto que a Folha 1 , mais nova, não apresentou diferença, enquanto a Folha 2, por ser mais velha, apresentou diferença em sua coloração, provavelmente pelos nutrientes móveis, como o nitrogênio, que foram das folhas mais velhas para as folhas mais novas.

De modo geral, é possível perceber que para a altura de plantas (Tabela 2), o uso do lodo de curtume promoveu um retardamento, podendo isso ser algo positivo, uma vez que muitas dessas plantas são colocadas em vasos e são desejadas que permaneçam pequenas pelo maior período possível, a fim de manterse adequada ao seu ambiente, pois se crescerem muito é possível que tenham que ser descartadas ou replantadas em outro local maior. Com isso, o lodo de curtume pode servir como um retardador de desenvolvimento para esse tipo de planta, visto que ele proporciona um crescimento lento.

\section{CONCLUSÃO}

A utilização de lodo de curtume na proporção de $10 \%$ mostrou-se eficiente no ganho das características de desenvolvimento, visto que foi igual ao tratamento convencional em todas as caracteristicas, exeto em altura de planta, podendo ser uma alternativa o uso deste material nesta proporção.

Para as características fisiológicas, é observado que a utilização de lodo de curtume apresentou diferença somente no índice de flavonóides e no balanço de nitrogênio quando comparado ao tratamento convencional.

\section{REFERÊNCIAS}

ASMUSSEN-LANGE, C. B.; MAUNDER, M.; FAY, M. F. Conservation genetics of the critically endangered Round Island bottle palm, Hyophorbela genicaulis (Arecaceae): can cultivated stocks supplement a residual wild population?. Botanical Journal of the Linnean Society, v. 167, n. 3, p. 301-310, 2011.

BELTRÃO, N. E. M.; OLIVEIRA, M. I. P. Ecofisiologia das culturas de algodão, amendoim, gergelim, mamona, pinhão-manso e sisal. Brasília, EMBRAPA, p. 257-298, 2011. 
BERILLI, S. S.; QUIUQUI, J. P. C.; REMBINSKI, J.; SALLA, P. H. H.; BERILLI, A. P. C. G.; LOUZADA, J. M. Utilização de lodo de curtume como substrato alternativo para produção de mudas de café conilon. Coffee Science, v. 9, n. 4, p. 472-479, 2014.

BERILLI, S. S.; BERILLI, A. P. C. G.; CARVALHO, A. J. C.; FREITAS, S. J.; CUNHA, M.; FONTES, P. S. F. Níveis de cromo em mudas de café conilon desenvolvidas em substrato com lodo de curtume como adubação alternativa. Coffee Science, v. 10, n. 3, p. 320-328, 2015.

BERILLI, S. S.; BERILI, A. P. C. G.; LEITE, M. C. T.; QUARTEZANI, W. Z.; ALMEIDA, R. F.; SALES, R. A. Uso de Resíduos na Agricultura. In: NICOLI, CF; MONHOL C; MARQUES JUNIOR, E; et al. (Org.). AGRONOMIA: Colhendo as Safras do Conhecimento. 1oed. Alegre: CAUFES, 2017, v. 1, p. $10-38$.

BERILLI, S. S.; MARTINELI, L.; FERRAZ, T. M.; FIGUEIREDO, F. A. M. M. A.; RODRIGUES, W. P.; BERILLI, A. P. C. G; SALES, R. A.; FREITAS, S. J. Substrate Stabilization Using Humus with Tannery Sludge in Conilon Coffee Seedlings. Journal of Experimental Agriculture International, v. 21, n. 1, p. 1-10, 2018.

BRAHM, R. Ü.; MEDEIROS, C. A. B.; CARDOSO, J. H.; REISSER JUNIOR, C. Avaliação do efeito de diferentes substratos sobre o desenvolvimento de Euterpe Edulis (Mart.) e Roystonea regia (Kunth). Revista Brasileira de Agroecologia, v. 8, n. 2, p. 148-160, 2013.

BORIN, A. L. D. C.; FERREIRA, G. B.; CARVALHO, M. C. S.; FERREIRA, A. C. B., BOGIANI, J. C. Diagnose Visual de Deficiências Nutricionais do Algodoeiro. Embrapa Algodão-Circular Técnica 134 (INFOTECA-E), p.1-13, 2013

DRANSFIELD, J.; UHL, N. W.; ASMUSSEN, C. B.; BAKER, W. J.; HARLEY, M. M.; LEWIS, C. E. Genera Palmarum, the evolution and classification of palms. Richmond: Royal Botanic Gardens, Kew, 2008.
EMBRAPA. Sistema brasileiro de classificação de Solos. 3. ed. Rio de Janeiro: Embrapa Solos, 2013.

KANDIL, F. E.; GRACE, M. H.; SEIGLER, D. S.; CHEESEMAN, J. M. Polyphenolics in Rhizophora mangle L. leaves and their changes during leaf development and senescence. Trees, v. 18, p. 518-528, 2004.

Ministério de Agricultura, Pecuária e Abastecimento (MAPA). Instrução Normativa ñ 27, 05 de junho de 2006. Diário Oficial da União, Brasília, DF, 2006. Seção 1, Página 15.

MARTINS FILHO, S.; FERREIRA, A.; ANDRADE, B. S.; RANGEL, R. M.; SILVA, M. F. Diferentes substratos afetando o desenvolvimento de mudas de palmeiras. Revista Ceres, v. 54, n. 311, p. 80-86, 2007.

MATTHES, L. A. F.; UZZO, R.P. Palmeiras ornamentais: produção e cultivo. Campinas: Fundag, 2010. p. 188.

NAZARIO, S. L. S.; MIOTTI, A. A.; SOUZA, D. Análise do lodo de curtume para aplicação como biofertilizante. In: IX congresso nacional de excelência em gestão, 2013, Rio de Janeiro, RJ, Anais Eletrônicos... Rio de Janeiro, 2013. Disponível em: $<$ http://www.excelenciaemgestao.org/Portals/2/d ocuments/cneg9/anais/T13_0597_3474.pdf> Acesso em: 20 de agosto de 2017.

QUARTEZANI, W. Z.; SALES, R. A.; PLETSCH, T. A.; SILVA BERILLI, S. A.; NASCIMENTO, A. L.; HELL, L. R.; MANTOANELLI, E.; BERILLI, A. P. C. G; SILVA, R. T. P.; TOSO, R. Conilon plant growth response to sources of organic matter. African Journal of Agricultural Research, v. 13, n. 4, p. 181-188, 2018.

R Core Team. R: A language and environment for statistical computing. R Foundation for Statistical Computing, Vienna, Austria, 2016. Disponível em <http://www.Rproject.org/>. Acesso em 12 de junho de 2017. 
SALES, R. A.; AMBROZIM, C. S.; VITÓRIA, Y. T.; SALES, R. A.; BERILLI, S. S. Influência de diferentes fontes de matéria orgânica no substrato de mudas de Passiflora Morifolia. Enciclopédia Biosfera, v. 13, n. 24, p. 606-6015, 2016.

SALES, R. A.; SALES, R. A.; NASCIMENTO, T. A.; SILVA, T. A.; BERILLI, S. S.; SANTOS, R. A. Influência de diferentes fontes de matéria orgânica na propagação da Schinus Terebinthifolius Raddi. Scientia Agraria, v. 18, n. 4, p. 99-106, 2017.

SILVA, F. A. M.; SOUZA, I. V.; ZANON, J. A.; NUNES, G. M.; SILVA, R. B.; FERRARI, S. Produção de mudas de juçara com resíduos agroindustriais e lodo de esgoto compostados. Revista Brasileira de Engenharia de Biossistemas, v. 9, n. 2, p. 109-121, 2015.

SOARES, A. N. R.; ROCHA JÚNIOR, V. F.; VITÓRIA, M. F.; SILVA, A. V. C. Germinação de sementes de nim em função da maturidade fisiológica e do substrato. Nucleus, v. 13, n. 1, p. 215-222, 2016. 